GANIT J. Bangladesh Math. Soc. (ISSN 1606-3694) 39 (2019) 71-85

DOI: https://doi.org/10.3329/ganit.v39i0.44159

\title{
RIEMANNIAN GEOMETRY AND MODERN DEVELOPMENTS
}

\author{
A. K. M. Nazimuddin ${ }^{1, *}$ and Md. Showkat $\mathrm{Ali}^{2}$ \\ ${ }^{1}$ Department of Mathematical and Physical Sciences, East West University, Dhaka-1212 \\ ${ }^{2}$ Department of Applied Mathematics, University of Dhaka, Dhaka-1000 \\ *Corresponding author: nazimuddin@ewubd.edu
}

Received : 28-04-2018

Accepted : 15-04-2019

\begin{abstract}
In this paper, we compute the Christoffel Symbols of the first kind, Christoffel Symbols of the second kind, Geodesics, Riemann Christoffel tensor, Ricci tensor and Scalar curvature from a metric which plays a fundamental role in the Riemannian geometry and modern differential geometry, where we consider MATLAB as a software tool for this implementation method. Also we have shown that, locally, any Riemannian 3-dimensional metric can be deformed along a directioninto another metricthat is conformal to a metric of constant curvature.
\end{abstract}

Keywords: Riemannian Geometry, Computational Method, Flat Deformation.

\section{Introduction}

The purpose of this section is to discuss an implementation method on n-dimensional Riemannian manifolds using a computer technique. A Riemannian manifold is a differentiable manifold in which each tangent space is equipped with an inner product $\langle\cdot, \cdot\rangle$ in a manner which varies smoothly from point to point. All differentiable manifolds (of constant dimension) can be given the structure of a Riemannian manifold. Geodesics plays an important role in many applications, especially in nuclear physics, image processing. OvidiuCalin and Vittorio Mangione [1] considered the Heisenberg manifold structure to provide a qualitative characterization for geodesics under nonholonomic constraints. Our implementation approach can successfully well illustrate the important parameters such as Christoffel coefficients that are required in the determination of tensors. This symbol appears in many calculations in Geometry where we use non-Cartesian coordinates. In $\mathrm{n}$-dimensions it has a total of $n^{3}$ components. Thus, whereas it is easy to compute this symbol in 2 or 3 dimensions, it becomes highly tedious to evaluate components of the Christoffel symbols in higher dimensions but it is quite an easy task to deal with such situations if one can use algebraic computations for this purpose. However, it is not always possible to have the readymade routines available that can be used in situations like this. Thus, it is of great use if one can write small routines to algebraically compute such expressions.

(C) GANIT: Journal of Bangladesh Mathematical Society, 2019 


\section{Riemannian Metrics and Levi-Civita Connection}

Let $M$ be a smooth manifold. A bilinear symmetric positive-definite form

$$
g_{p}: T_{p} M \times T_{p} M \rightarrow \mathbb{R}
$$

defined for every $p \in M$ and smoothly depending on $\mathrm{p}$ is called a Riemannian metric on $M$. Positive-definite means that $g_{p}(v, v)>0$ for every $v=0, v \in T_{p} M$. Smoothly depending on $p$ means that for every pair $X_{p}, Y_{p}$ of $\mathbb{C}^{\infty}$ smooth vector fields on $M$ the expression $g_{p}\left(X_{p}, Y_{p}\right)$ defines a $\mathbb{C}^{\infty}$-smooth function of $p \in M$.

Alternatively, consider a coordinateneighbourhood on $M$ containing $p$ and let $x^{i}, i=$ $1, \ldots, \operatorname{dim} M$ be the local coordinates. Then any two tangent vectors $u, v \in T_{p} M$ may be written as $u=u^{i}\left(\partial / \partial x^{i}\right)_{p}, v=v^{i}\left(\partial / \partial x^{i}\right)_{p}$ and $g_{p}(u, v)=g_{i j}(p) u^{i} v^{j}$ where the functions $g_{i j}(p)=$ $g\left(\left(\partial / \partial x^{i}\right)_{p},\left(\partial / \partial x^{i}\right)_{p}\right)$ express the coefficients of the metric $g$ in local co-ordinates. One often uses the following notation for a metric in local coordinates $g=g_{i j} d x^{i} d x^{j}$. The bilinear form (metric) $g$ will be smooth if and only if the local coefficients $g_{i j}=g_{i j}(x)$ are smooth functions of local coordinates $x^{i}$ on each coordinate neighbourhood.

Theorem 2.1. Any smooth manifold $M$ can be given a Riemannian metric. [5]

Definition 2.1. A connection on a manifold $M$ is a connection on its tangent bundle TM. A choice of local coordinates $x$ on $M$ determines a choice of local trivialization of $T M$ (using the basis vector fields $\frac{\partial}{\partial x^{i}}$ on coordinate patches). The transition function $\emptyset$ for two trivializations of $T M$ is given by the Jacobi matrices of the corresponding change of coordinates $\left(\varnothing_{p}^{i}\right)=\left(\frac{\partial x^{i}}{\partial x^{p}}\right)$.

Let $\Gamma_{j k}^{i}$ be the coefficients (Christoffel symbols) of a connection on $M$ in local coordinates $x^{i}$. For any other choice $x^{p}$ of local coordinates the transition law on the overlap becomes

$$
\Gamma_{j k}^{i}=\Gamma_{q r}^{p} \frac{\partial x^{i}}{\partial x^{p}} \frac{\partial x^{q}}{\partial x^{j}} \frac{\partial x^{r}}{\partial x^{k}}+\frac{\partial x^{i}}{\partial x^{p}} \frac{\partial^{2} x^{p}}{\partial x^{j} \partial x^{k}}
$$

One can see from the above formula that if $\Gamma_{j k}^{i}$ are the coefficients of a connection on $M$ then $\Gamma_{j k}^{i}$ also are the coefficients of some well-defined connection on $M$ (in general, this would be a different connection). The difference $T_{j k}^{i}=\Gamma_{j k}^{i}-\Gamma_{k j}^{i}$ is called the torsion of a connection $\left(\Gamma_{j k}^{i}\right)$. The transformation law for $T_{j k}^{i}$ is

$$
T_{j k}^{i}=T_{q r}^{p} \frac{\partial x^{i}}{\partial x^{p}} \frac{\partial x^{q}}{\partial x^{j}} \frac{\partial x^{r}}{\partial x^{k}}
$$

thus the torsion of a connection is a well-defined anti-symmetric bilinear map sending a pair of vector fields $X, Y$ to a vector field $T(X, Y)=T_{j k}^{i} X^{j} Y^{k}$ on $M$.

Theorem 2.2. On any Riemannian manifold $(M, g)$ there exists a unique connection $D$ such that

(1) $d(g(X, Y))(Z)=g\left(D_{Z} X, Y\right)+g\left(X, D_{Z} Y\right)$ for any vector fields $X, Y, Z$ on $M$; and

(2) the connection $D$ is symmetric, where $D$ is called the Levi-Civita connection of the metric $g$. 
The condition (1) in the above theorem is sometimes written more neatly as

$$
d g(X, Y)=g(D X, Y)+g(X, D Y) .
$$

\section{Geodesics on a Riemannian Manifold}

Let $\mathrm{E} \rightarrow \mathrm{M}$ be a vector bundle endowed with a connection $\left(\Gamma_{j k}^{i}\right)$. A parameterized smooth curve on the base $M$ may be written in local coordinates by $x^{i}(t)$. A lift of this curve to E is locally expressed as $\left(x^{i}(t), a^{j}(t)\right)$ using local trivialization of the bundle $\mathrm{E}$ to define coordinates $a^{j}$ along the fibres. A tangent vector $(\dot{x}(t), \dot{a}(t)) \in T_{\left(x^{i}(t), a^{j}(t)\right)} E$ to a lifted curve will be horizontal at every t precisely when $a(t)$ satisfies a linear ODE

$$
\dot{a}^{i}+\Gamma_{j k}^{i}(x) a^{j} \dot{x}^{k}=0
$$

where $i, j=1, \ldots, \operatorname{rank} E, k=1, \ldots, \operatorname{dim} B$. Now if $E=T M$ then there is also a canonical lift of any smooth curve $\gamma(t)$ on the base, as $\dot{\gamma}(t) \in T_{\gamma(t)} M$.

Definition 3.1. A curve $\gamma(t)$ on a Riemannian manifold $M$ is called a geodesic if $\dot{\gamma}(t)$ at every $t$ is horizontal with respect to the Levi-Civita connection. The condition for a path in $M$ to be a geodesic may be written explicitly in local coordinates as

$$
\ddot{x}^{i}+\Gamma_{j k}^{i}(x) \dot{x}^{j} \dot{x}^{k}=0
$$

a non-linear second-order ordinary differential equation for a path $x(t)=\left(x^{i}(t)\right)$ (here $i, j, k=$ $1, \ldots, \operatorname{dim} M)$. By the basic existence and uniqueness theorem from the theory of ordinary differential equations, it follows that for any choice of the initial conditions $x(0)=p, \dot{x}(t)=a$ there is a unique solution path $x(t)$ defined for $|t|<\varepsilon$ for some positive $\varepsilon$. Thus for any $p \in M$ and $a \in T_{p} M$ there is a uniquely determined (at least for any small $|t|$ ) geodesic with this initial data (i.e. 'coming out of $p$ in the direction $a$ ').

Proposition 3.1. If $\gamma(t)$ is a geodesic on $(M, g)$ then $|\dot{\gamma}(t)| g=$ constant.

\section{Curvature of a Riemannian Manifold}

Let $g$ be a metric on a manifold M. The (full) Riemann curvature $R=R(g)$ of $g$ is, by definition, the curvature of the Levi-Civita connection of $g$. Thus $R \in \Omega_{M}^{2}(E n d(T M))$, locally a matrix of differential 2-forms $R=\frac{1}{2}\left(R_{j, k l}^{i} d x^{l} \wedge d x^{k}\right), i, j, k, l=1 \ldots n=\operatorname{dim} M$. The coefficients $\left(R_{j, k l}^{i}\right)$ form the Riemann curvature tensor of $(M, g)$. Given two vector fields, $Y$, one can form an endomorphism field $R(X, Y) \in \Gamma(E n d(T M))$; its matrix in local coordinates is $R(X, Y)_{j}^{i}=$ $R_{j, k l}^{i} X^{k} Y^{k}$ (as usual $X=X^{k} \partial_{k}, Y=Y^{l} \partial_{l}$ ). Denote $R_{k}=R(\partial k, \partial l) \in \operatorname{End}\left(T_{p} M\right)$ (here $p$ is any point in the coordinate neighbourhood). In local coordinates a connection (covariant derivative) may be written as $d+A$, with $A=\Gamma_{j k}^{i} d x^{k}=A_{k} d x^{k}$. We write

$$
D_{k}=D_{\frac{\partial}{\partial x_{k}}}=\frac{\partial}{\partial x_{k}}+A_{k} \text {. }
$$


The definition of the curvature form of a connection yields an expression in local coordinates

$$
R_{j, k l}^{i}=\left(D_{l} D_{k} \frac{\partial}{\partial x^{j}}-D_{k} D_{l} \frac{\partial}{\partial x^{j}}\right)^{i}, \text { or } R_{k l}=-\left[D_{k}, D_{l}\right]
$$

considering the coefficient at $d x^{l} \wedge d x^{k}$. Now $D_{X}=X^{k} D_{k}$. So we have

$$
\begin{aligned}
-\left[D_{X}, D_{Y}\right] & =-\left[X^{k} D_{k}, X^{l} D_{l}\right]=-X^{k}\left(\partial_{k} Y^{l}\right) D_{l}-X^{k} Y^{l} D_{k} D_{l}+Y^{k}\left(\partial_{k} X^{l}\right) D_{l}+X^{k} Y^{l} D_{l} D_{k} \\
& =X^{k} Y^{l} R_{k l}-[X, Y]^{l} D_{l}
\end{aligned}
$$

We have thus proved.

\section{Proposition 4.1.}

(i) $R_{i j, k l}=-R_{i j, l k}=R_{j i, l k}$ (ii) $R_{j, k l}^{i}+R_{k, l j}^{i}+R_{l, j k}^{i}=0$ (iii) $R_{i j, k l}=R_{k l, i j}$

Proof. (i) The first equality is clear. For the second equality, one has, from the definition of the Levi-Civita connection, $\frac{\partial g_{k l}}{\partial x^{i}}=g\left(D_{i} \frac{\partial}{\partial x^{k}}, \frac{\partial}{\partial x^{l}}\right)+g\left(\frac{\partial}{\partial x^{k}}, D_{i} \frac{\partial}{\partial x^{l}}\right)$ and further $\frac{\partial^{2} g_{k l}}{\partial x^{j} \partial x^{i}}=g\left(D_{j} D_{i} \frac{\partial}{\partial x^{k}}, \frac{\partial}{\partial x^{l}}\right)+g\left(D_{i} \frac{\partial}{\partial x^{k}}, D_{j} \frac{\partial}{\partial x^{l}}\right)+g\left(D_{j} \frac{\partial}{\partial x^{k}}, D_{i} \frac{\partial}{\partial x^{l}}\right)+g\left(\frac{\partial}{\partial x^{k}}, D_{j} D_{i} \frac{\partial}{\partial x^{l}}\right)$

The right-hand side of the above expression is symmetric in $i, j$ as $\frac{\partial^{2} g_{k l}}{\partial x^{j} \partial x^{i}}=\frac{\partial^{2} g_{k l}}{\partial x^{i} \partial x^{j}}$. The antisymmetric part of the right-hand side (which has to be zero) equals $R_{i j, k l}+R_{j i, k l}$.

(ii) Firstly, $\left(D_{k} \frac{\partial}{\partial x^{j}}\right)^{i}=\Gamma_{j k}^{i}=\left(D_{j} \frac{\partial}{\partial x^{k}}\right)^{i}$, by the symmetric property of the Levi-Civita. The claim now follows by straightforward computation.

(iii) Multiplying (ii) by $g_{i q}$ gives $R_{i j, k l}+R_{i k, l j}+R_{i l, j k}=0$ similarly $R_{j k, l i}+R_{j l, i k}+R_{j i, k l}=$ $0 R_{k l, i j}+R_{k i, j l}+R_{k j, l i}=0$ and $R_{l i, j k}+R_{l j, k i}+R_{l k, i j}=0$

Adding up the four identities and making cancellations using (i) (the 'octahedron trick') gives the required result.

There are natural ways to extract "simpler" quantities (i.e. with less components) from the Riemann curvature tensor.

Definition 4.1. The Ricci curvature of a metric $g$ at a point $p \in M, \operatorname{Ric} C_{P}=\operatorname{Ric}(g)_{p}$, is the trace of the endomorphism $v \rightarrow R_{p}(x, v) y$ of $T_{p} M$ depending on a pair of tangent vectors $x, y \in T_{p} M$. Thus in local coordinates $\operatorname{Ric}(p)$ is expressed as a matrix $\operatorname{Ric}=\left(\operatorname{Ric}_{i j}\right), \operatorname{Ric}_{i j}=\sum_{q} R_{i, j q}^{q}$. That is, the Ricci curvature at $p$ is a bilinear form on $T_{p} M$. A consequence of Proposition4.2 (iii) is that this bilinear form is symmetric, $R i c_{i j}=R i c_{j i}$.

Definition 4.2. The scalar curvature of a metric $g$ at a point $p \in M, s=\operatorname{scal}(g)_{p}$ is a smooth function on $M$ obtained by taking the trace of the bilinear form $R i c_{i j}$ with respect to the metric $g$.

If local coordinates are chosen so that $g_{i j}(p)=\delta i j$, then the latter definition means that $s(p)=$ $\sum_{i} R_{i i}(p)=\sum_{i, j} R_{i j, j i}(p)$. For a general $g_{i j}$, the formula may be written as $s=\sum_{i} g^{i j} R i c_{i j}$, where $g^{i j}$ is the induced inner product on the cotangent space with respect to the dual basis, algebraically $\left(g^{i j}\right)$ is the inverse matrix of $\left(g_{i j}\right)$. 


\section{Computer Code}

In the current section, we have presented our developed computer code with an example. We have developed this code by using a mathematical programming language MATLAB [8].

Example 5.1. Consider the metric for the three-sphere in coordinates $x^{\mu}=(\psi, \theta, \varphi)$ is given by [2]

$$
d s^{2}=\mathrm{d} \psi^{2}+\sin ^{2} \psi\left(d \theta^{2}+\sin ^{2} \theta d \varphi^{2}\right)
$$

\section{MATLAB Code 1: (Calculating the Christoffel symbols of the first kind)}

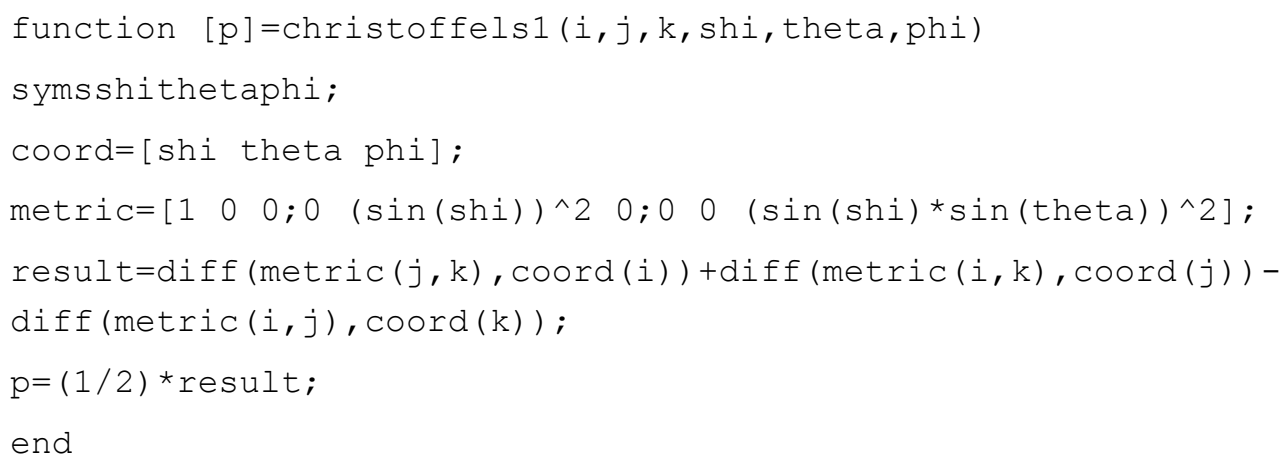

If we run the above code for a particular input, then we will get a corresponding output. The followings are nonvanishing components and all other components are zero.

Input: christoffels1 $(1,2,2)$

Output: $\cos (\operatorname{sh} i){ }^{*} \sin (\operatorname{sh} i)$

Input: christoffels1 $(1,3,3)$

Output: $\cos (\operatorname{sh} i) * \sin (\operatorname{sh} i) * \sin (\text { theta })^{\wedge} 2$

Input: christoffels1 $(2,1,2)$

Output: $\cos (\operatorname{sh} i) * \sin (\operatorname{sh} i)$

Input: christoffels1 $(2,2,1)$

Output: $-\cos (\operatorname{sh} i){ }^{*} \sin (\operatorname{sh} i)$

Input: christoffels1 $(2,3,3)$

Output: $\cos \left(\right.$ theta) ${ }^{*} \sin (\operatorname{sh} i)^{\wedge} 2 * \sin ($ theta)

Input: christoffels1 $(3,1,3)$

Output: $\cos (\operatorname{sh} i){ }^{*} \sin (\operatorname{sh} i) * \sin ($ theta $) \wedge 2$

Input: christoffels1 $(3,2,3)$

Output: $\cos \left(\right.$ theta) ${ }^{*} \sin (\operatorname{sh} i)^{\wedge} 2{ }^{*} \sin ($ theta)

Input: christoffels1 $(3,3,1)$ 
Output:- $\cos (\operatorname{sh} i) * \sin (\operatorname{sh} i) * \sin (\text { theta })^{\wedge} 2$

Input: christoffels1 $(3,3,2)$

Output: $-\cos \left(\right.$ theta) ${ }^{*} \sin (\operatorname{sh} i)^{\wedge} 2 * \sin ($ theta)

\section{MATLAB Code 2: (Calculating the Christoffel symbols of the second kind)}

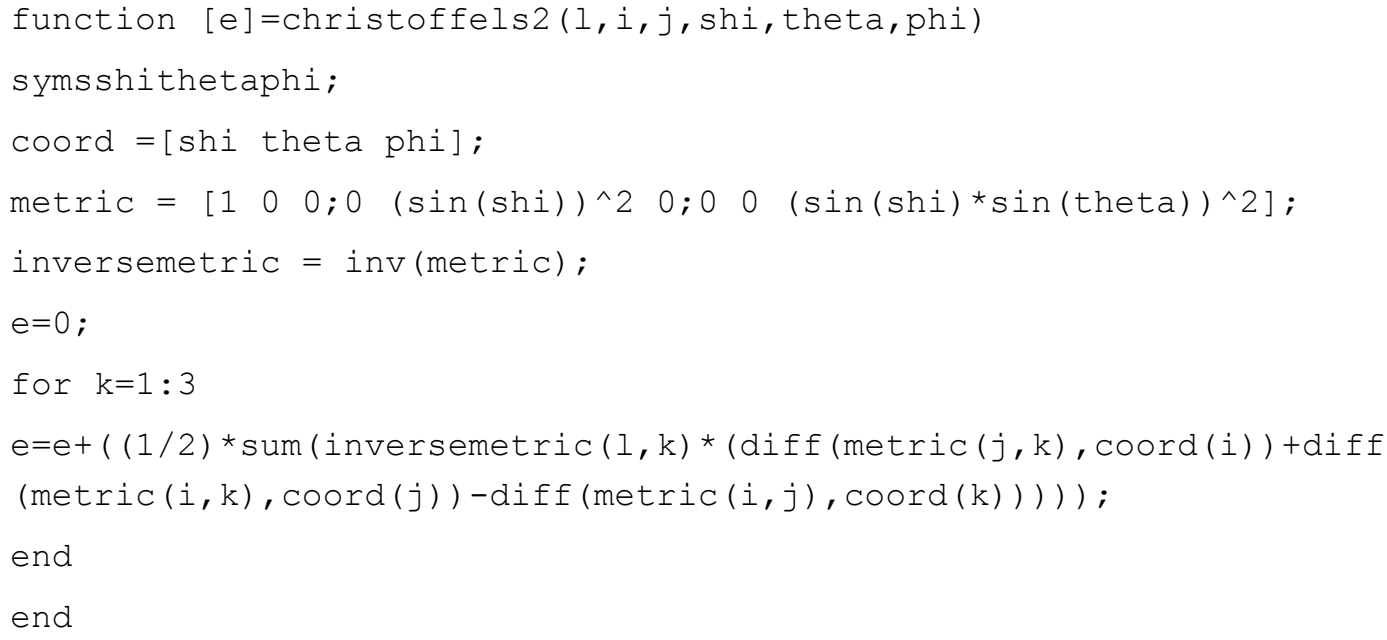

If we run the above code for a particular input, then we will get a corresponding output. The followings are nonvanishing components and all other components are zero.

Input: christoffels2 $(1,2,2)$

Output: $-\sin (\operatorname{sh} i){ }^{*} \cos (\operatorname{sh} i)$

Input: christoffels2 $(1,3,3)$

Output: $-\sin (\operatorname{sh} i){ }^{*} \cos (\operatorname{sh} i){ }^{*} \sin ($ theta $) \wedge 2$

Input: christoffels2 $(2,1,2)$

Output: cos (shi)/sin (shi)

Input: christoffels2 $(2,2,1)$

Output: $\cos (\operatorname{sh} i) / \sin (\operatorname{sh} i)$

Input: christoffels2 $(2,3,3)$

Output: - cos (theta)*sin (theta)

Input: christoffels2 $(3,1,3)$

Output: cos (shi)/sin (shi)

Input: christoffels2 $(3,2,3)$

Output: cos (theta)/sin (theta)

Input: christoffels2 $(3,3,1)$ 
Output: $\cos (\operatorname{sh} i) / \sin (\operatorname{sh} i)$

Input: christoffels2 $(3,3,2)$

Output: $\cos ($ theta)/sin(theta)

\section{MATLAB Code 3: (Calculating the geodesic)}

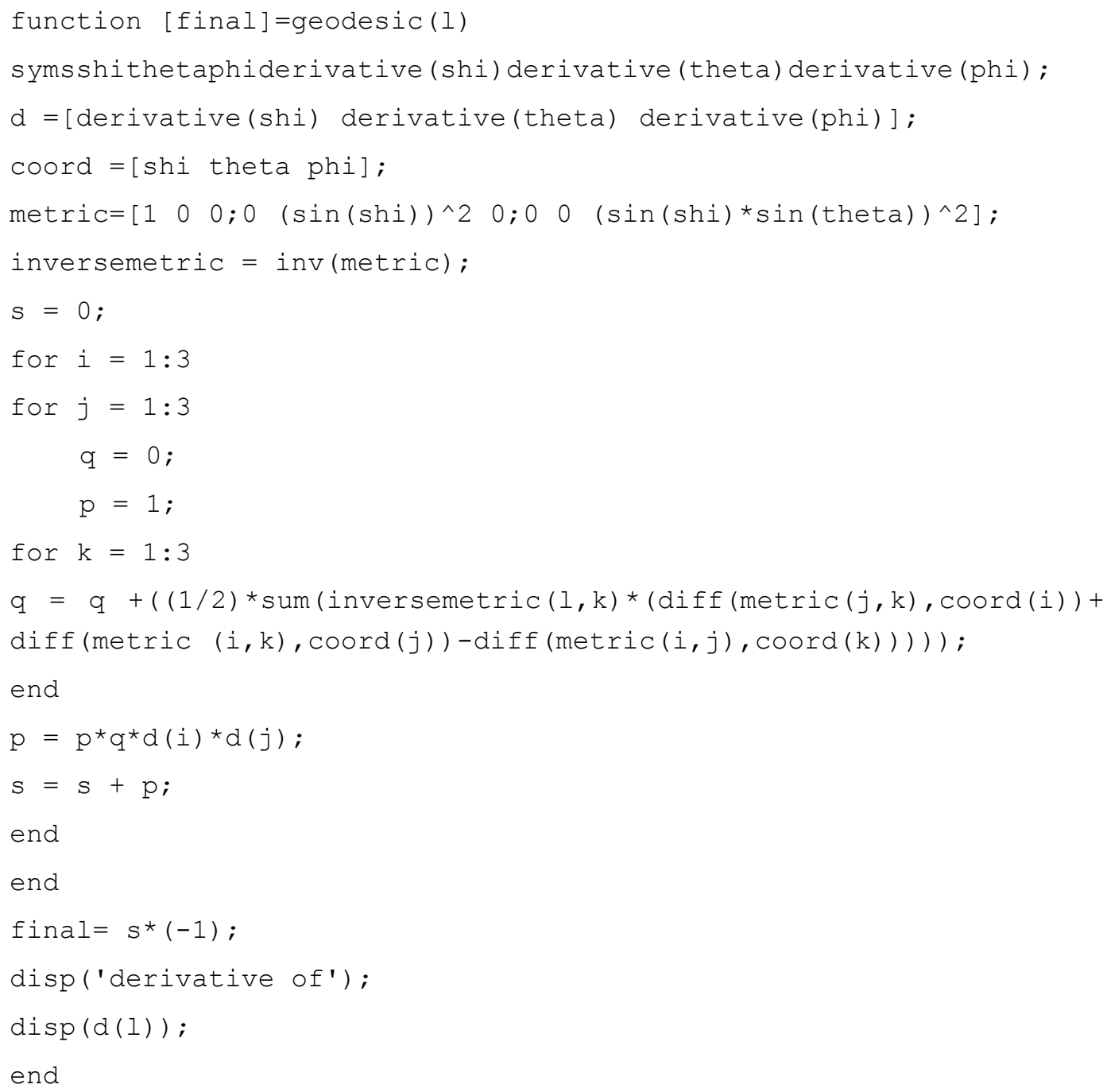

If we run the above code for a particular input, then we will get a corresponding output.

Input: geodesic ( 1 )

Output: derivative of derivative $(\operatorname{sh} i)=\cos (\operatorname{sh} i){ }^{*} \sin (\operatorname{sh} i) * \operatorname{derivative}($ phi $) \wedge 2 * \sin (\text { theta })^{\wedge} 2$ $+\cos (\operatorname{sh} i) * \sin (\operatorname{sh} i) * \operatorname{derivative}($ theta)^2 
Input: geodesic (2)

Output: derivative of derivative $($ theta $)=$ derivative $(\text { phi })^{\wedge} 2 * \cos \left(\right.$ theta) ${ }^{*} \sin ($ theta $)-(2 * \cos ($ shi $) *$ derivative $($ shi) * derivative (theta))/sin(shi)

Input: geodesic (3)

Output: derivative of derivative $($ phi) $=$

- (2*derivative (phi)* cos (shi)*derivative (shi)) / sin (shi) - (2* derivative (phi)* cos (theta)*derivative(theta))/sin(theta)

\section{MATLAB Code 4: (Calculating the Riemann Christoffel tensor)}

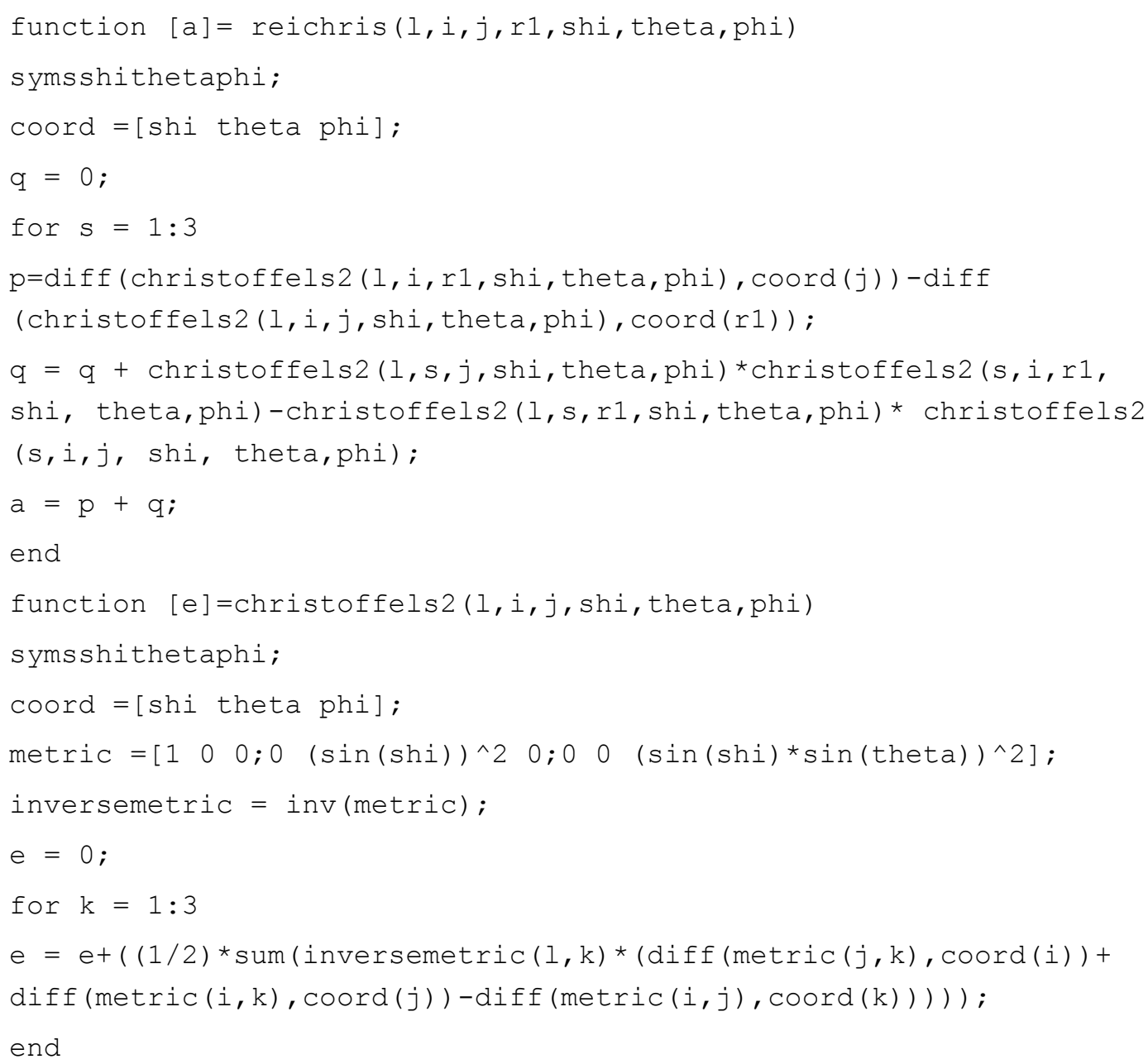


end

end

If we run the above code for a particular input, then we will get a corresponding output. The followings are nonvanishing components and all other components are zero or are related via symmetries.

Input: reichris $(1,2,1,2)$

Output: $\sin (\operatorname{sh} i)^{\wedge} 2$

Input: reichris $(1,3,1,3)$

Output: $\sin (\operatorname{sh} i)^{\wedge} 2 \star \sin (\text { theta })^{\wedge} 2$

Input: reichris $(2,3,2,3)$

Output: $\sin (\operatorname{sh} i)^{\wedge} 2 * \sin (\text { theta })^{\wedge} 2$

\section{MATLAB Code 5: (Calculating the Ricci tensor)}

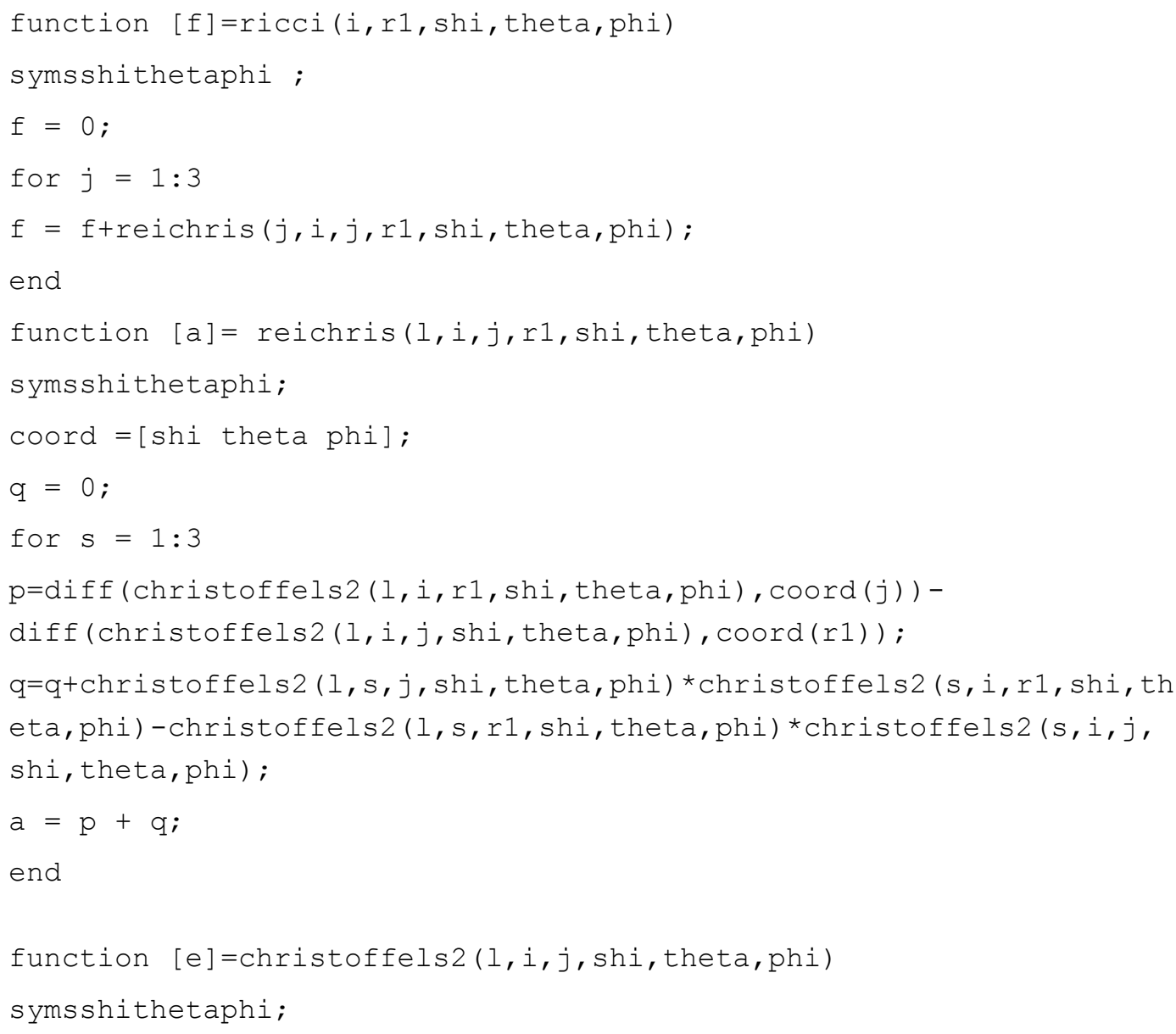




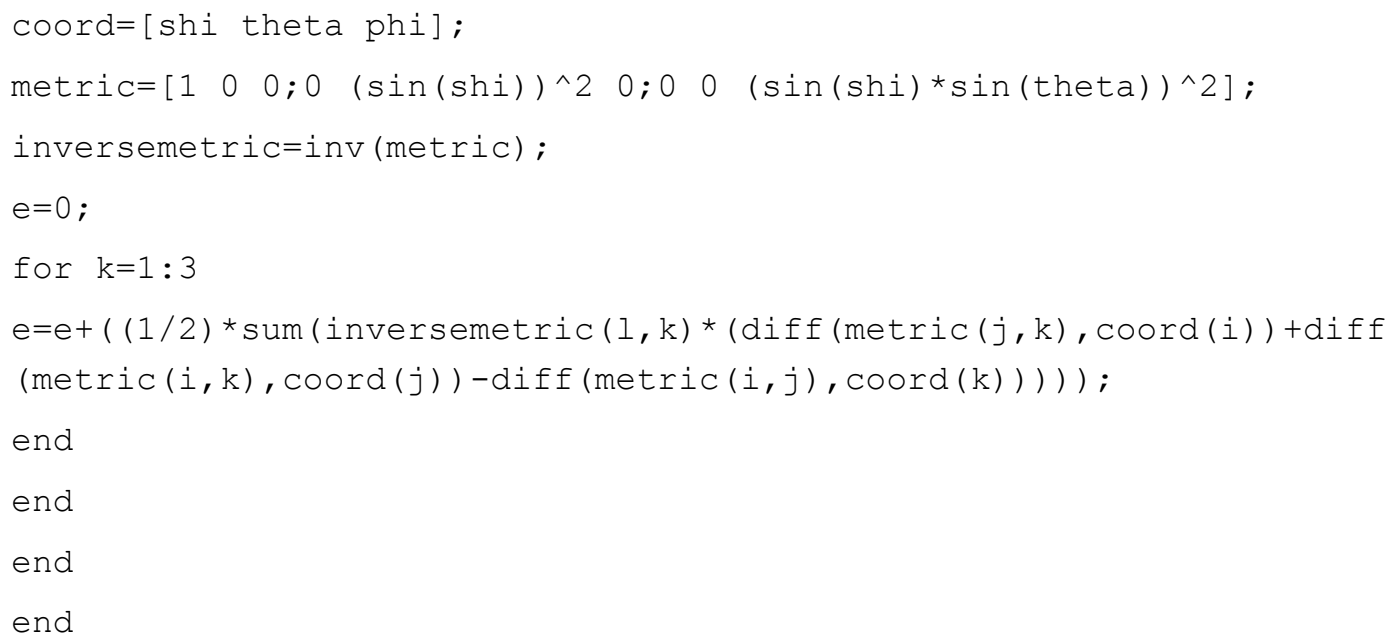

If we run the above code for a particular input, then we will get a corresponding output. The followings are nonvanishing components and all other components are zero.

Input: $\operatorname{ricci}(1,1)$

Output: 2

Input: $\operatorname{ricci}(2,2)$

Output: $2 \sin (\operatorname{sh} i)^{\wedge} 2$

Input: $\operatorname{ricci}(3,3)$

Output: $2 \sin (\operatorname{sh} i)^{\wedge} 2 * \sin (\text { theta })^{\wedge} 2$

\section{MATLAB Code 6: (Calculating the scalar curvature tensor)}

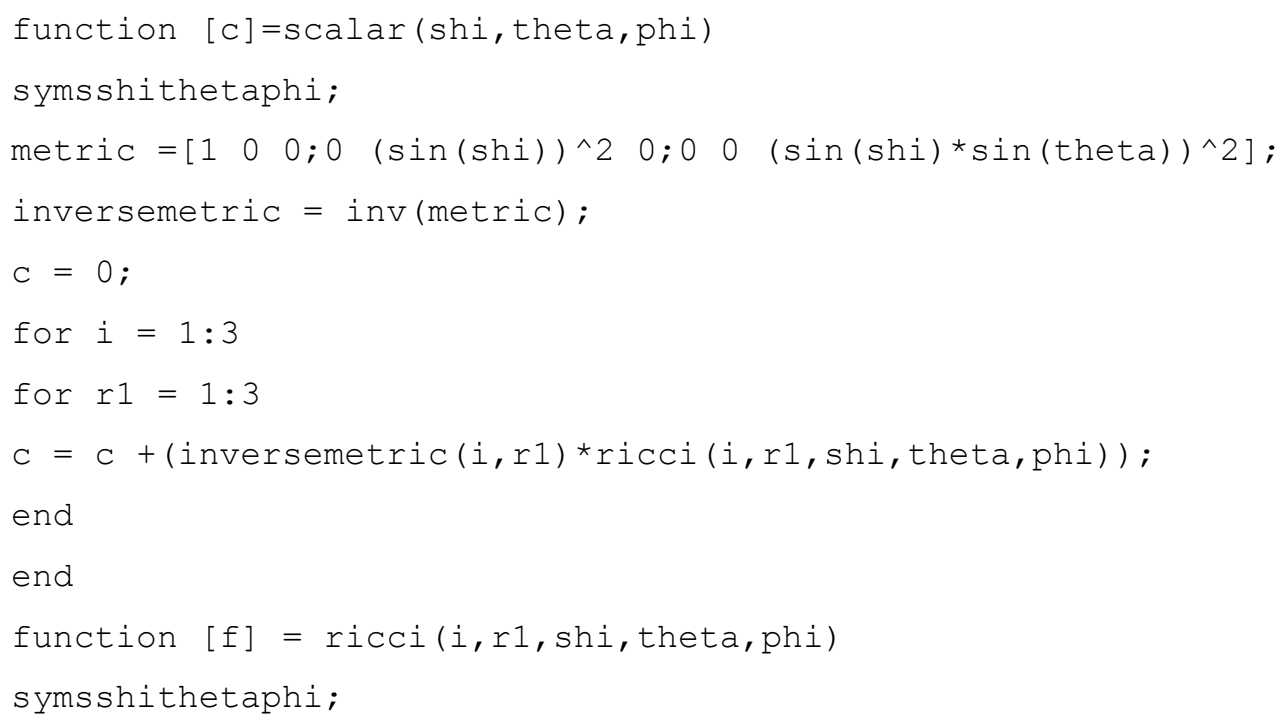




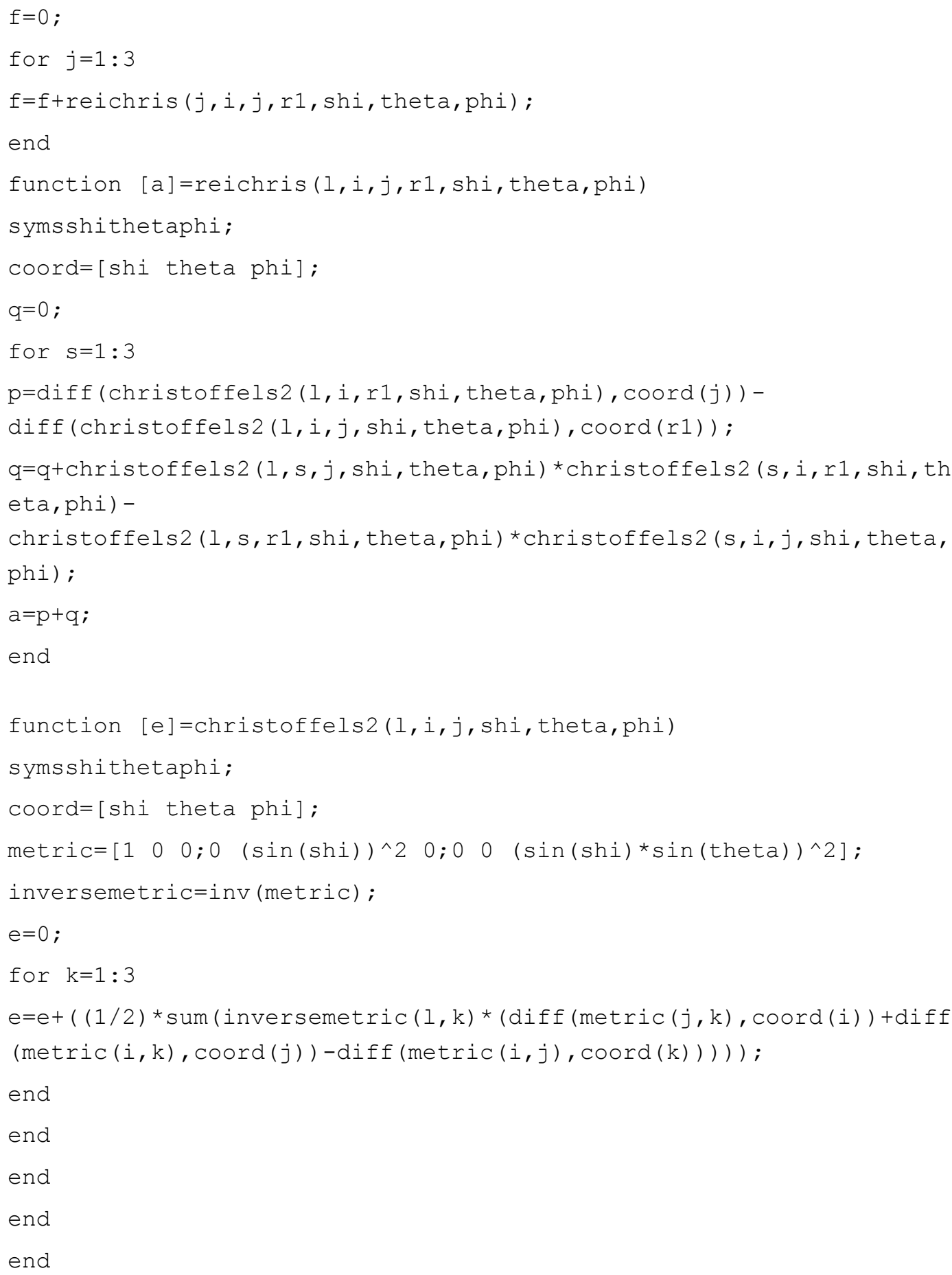

If we run the above code, then we will get the following output. 
Input: scalar

Output: 6

\section{Three-Dimensional Metrics as Deformations of a Constant Curvature Metric}

It is known, since an old result by Riemann, that a $n$-dimensional metric has $f=n(n-1) /$ 2 degrees of freedom, that is, it is locally equivalent to the giving of $f$ functions. As this feature is related to some particular choices of local charts, which are obviously non-geometric objects, it seems to be generically a not covariant property.

According to it, a two-dimensional metric has $f=1$ degrees of freedom. In this case, however, a stronger result holds, as it is well known [4], namely: any two-dimensional metric $\mathrm{g}$ is locally conformally flat, $=\varphi \eta, \varphi$ being the conformal deformation factor and $\eta$ the flat metric.

Contrarily to what the above Riemann's general result suggests, the two dimensional case is intrinsic and covariant, i.e. it only needs the knowledge of the metric $g$ and only involves tensor quantities, specifically, the sole degree of freedom is represented by a scalar, the conformal deformation factor $\varphi$. The question thus arises of, whether or not, for $n>2$ there exist similar intrinsic and covariant local relations between an arbitrary metric $g$, on the one hand, and the corresponding flat one $\eta$ together with a set of $f$ covariant quantities on the other. To our knowledge, no result of this type has been published. Indeed, the known results concerning the diagonalization of any three-dimensional metric do not belong to this type. As a matter of fact, besides the $f=3$ scalars and the (more or less implicit) flat metric, these results also involve a particular orthogonal triad of vector fields. Also, in the context of the General Theory of Relativity, such a $n$-dimensional relation has been proposed by one of us, but unfortunately it remains for the moment only a mere conjecture [3].

In this section we shall answer affirmatively the three-dimensional case. This dimension is the solution to the equation $f=n$, so that one is tempted to take (the components of) a vector field as the covariant set (of $f=3$ quantities). On the other hand, the result being deliberately local, it would seem that the essentials of the flat metric in this matter is its minimal freedom, i.e. the maximal dimension of its isometry group, so that it should be possible to substitute it by a prescribed constant curvature metric. We shall see that both assumptions work. In fact, this section is devoted to prove the following main result:

Theorem 6.1. Any three-dimensional metric gmay be locally obtained from a constant curvature metric, $h$, by a deformation like

$$
g=\sigma h+\varepsilon s \otimes s
$$

where $\sigma$ and sare respectively a scalar and a one-form, the sign $\varepsilon= \pm 1$ and a functional relation between $\sigma$ and the Riemannian norm of $s$ can be arbitrarily prescribed. 
This result should be interesting in geometrical as well as in physical situations. In geometry, perhaps one of the first questions to be answered is the following: In two dimensions it is known that the gauge of the conformal factor $\sigma$ or, equivalently, the set of flat metric tensors conformal to a given metric is given by the solutions of the Laplacian, $\Delta \sigma=0$ [7].

In classical physics, the above theorem should be useful in (finite) deformation theory of materials; equation (6.1) may be considered as an ideal universal deformation law, allowing, from an unconstrained or not initial state (described in material coordinates by the tensor $h$ ), to reach any other deformation state (described in the same coordinates by the tensor $g$ ). This ideal universal law allows to associate, to every deformation state of a material, a vector field $s$ among those of the gauge class of the flat metric.

In general relativity, any vacuum space-time is locally equivalent to its Cauchy data, $\{g, K\}, g$ being the spatial metric and $K$ the extrinsic curvature of the initial instant. These data have to verify the constraint equations, a set of four equations for which many years ago Lichnerowicz showed [6] that to every arbitrarily given metric $g^{\prime}$ it corresponds a unique solution $\{g, K\}$ such that $g=\sigma g^{\prime}$.This beautiful result is however useless for precise physical situations because, $g$ being initially unknown, one does not see how to choose the good starting metric $g^{\prime}$, which has to give $g$ by conformity. Such an objection may be eliminated using (6.1) in the constraint equations. Our theorem also allows to translate notions such as asymptotic flatness or spatial singularity in terms of the differential 1 -form sover a flat metric $h$.

\subsection{Flat Deformation of a Given Metric}

Instead of proving theorem 6.1 as stated in the introduction, we shall prove the following equivalent result:

Theorem 6.2. Let $(M, g)$ be a Riemannian 3-manifold. There locally exist a function $\varphi$ and a differential 1 -form $\mu$ such that the tensor

$$
g^{\prime}:=\varphi g-\varepsilon \mu \otimes \mu
$$

(with $\varepsilon= \pm 1$ ) is also a Riemannian metric with constant curvature. Besides, an arbitrary relation between $\varphi$ and $|\mu|^{2}=g^{i j} \mu_{i} \mu_{j}$ can be imposed in advance. The equivalence between both theorems follows immediately on substituting

$h=g^{\prime}, \sigma=\varphi^{-1}, \sigma=\varphi^{-\frac{1}{2}} \mu$ into equation (6.1). The present formulation (6.2) stresses that we seek to derive $g^{\prime}$ from a given $g$.

The proof is based on the comparison of the Riemannian geometries respectively defined by $g$ and $g^{\prime}$.

We start by considering the Riemannian connections $\Delta$ and $\Delta^{\prime}$. In an arbitrary frame $\left\{e_{i}\right\}_{i=1,2,3}$ the expression (6.2) reads:

$$
g^{\prime}{ }_{i j}:=\varphi g_{i j}-V_{i j} \text { with } V_{i j}:=\mu_{i} \mu_{j}
$$


We shall consider the difference tensor:

$$
B_{k i}^{j}:=\gamma_{k i}^{\prime j}-\gamma_{k i}^{j}(6.4)
$$

which is symmetric:

$$
B_{k i}^{j}:=B_{i k}^{j}
$$

because both connections are torsion free.

Now, since $\Delta_{k} g_{i j}=\Delta^{\prime}{ }_{k} g^{\prime}{ }_{i j}=0$ and taking (6.5) into account, we easily obtain that:

$$
B_{i k}^{j}=\frac{1}{2}\left[\varphi_{k} g_{i r}+\varphi_{i} g_{k r}-\varphi_{r} g_{i k}-\Delta_{k} M_{i r}-\Delta_{i} M_{k r}+\Delta_{r} M_{i k}\right] h^{\prime r j}
$$

where

$$
h^{\prime r j}:=\varphi^{-1}\left(g^{r j}+\frac{1}{\varphi-m_{0}} M^{r j}\right), \text { with } m_{0}:=g^{i j} M_{i j}=\varepsilon|\mu|^{2}
$$

is the inverse metric for $g^{\prime}{ }_{i j}$.

For the sake of illustration we shall consider an example of 3-dimensional Riemannian manifolds and locally deform them into flat metrics, in the sense stated in theorem 6.2.

Example 6.1. (Schwarzschild Space)

The title is a shortening for the space 3-manifold for Schwarzschild coordinates in Schwarzschild spacetime. The metric is:

$\tilde{g}=k^{-1} d r \otimes d r+r^{2} d \theta \otimes d \theta+r^{2} \sin ^{2} \theta d \varphi \otimes d \varphi$

with $=1-\frac{2 m}{r}$, in the region $r>2 m$ (otherwise the metric is not Riemannian). This metric can be deformed into a flat metric in several ways. Among others: (A) Choosing $s=\sqrt{k^{-1}-1} d r$, we readily obtain:

$$
\tilde{g}=g^{\prime}+s \otimes s
$$

where $g^{\prime}=d r \otimes d r+r^{2} d \theta \otimes d \theta+r^{2} \sin ^{2} \theta d \varphi \otimes d \varphi$ is flat.

(B) It is well known that changing $r$ into the coordinate

$$
R=\frac{1}{2}(r \sqrt{k}+r-m), \quad r=R\left(1+\frac{m}{2 R}\right)^{2}
$$

the metric becomes: $\tilde{g}=\sigma g^{\prime}$, where

$$
\sigma=\left(1+\frac{m}{2 R}\right)^{4}, \quad g^{\prime}:=d R \otimes d R+R^{2} d \theta \otimes d \theta+R^{2} \sin ^{2} \theta d \varphi \otimes d \varphi
$$

is a flat metric.We have shown that, locally, any Riemannian 3-dimensional metric $g$ can be deformed along a direction sinto a metric $\sigma h$ that is conformal to a metric of constant curvature, as stated in theorem 6.1 .

\section{Conclusion}


Whereas algebraic softwares have made many lengthy and tedious calculations possible through various packages, it is quite difficult to write one's own codes without having much of a programming skill. It is interesting to note that despite being extremely simple, the described routine is quite powerful in its own right as it can evaluate Christoffel symbols, geodesics, Ricci tensor, Riemannian Christoffel tensor, Scalar curvature in any coordinated system in any arbitrary dimension. Also deformations of a metric of constant curvature is very important in Riemannian geometry where the direction sis not uniquely determined by the metric $g$, and the decomposition (6.1) can be achieved in an infinite number of ways. Determining more precisely the class of $\sigma$ and $s$ which deform a given ginto a constant curvature metric $h$ will be the object of future work.

Specially the case where both, $g$ and $h$ are flat.

\section{REFERENCES}

[1] Calin O. and Mangione V., "Geodesics with Constraints Heisenberg Manifolds," Results in Mathematics, 2(2003), 44-53.

[2] Carroll S. M. ,Spacetime and Geometry: An Introduction to General Relativity, 2003.

[3] Carroll S. M. ,Spacetime and Geometry: An Introduction to General Relativity, 2003.

[4] Eisenhart, L. P., A Treatise on the Differential Geometry of Curves and Surfaces, Dover Publications, Inc. New York, 1960.

[5] Gallot S., Hulin D. and Lafontaine J., 'Riemannian Geometry', Springer-Verlag, Berlin, Heidelberg, New York, 1987.

[6] Lichnerowicz, A., J. Math. Pures Appl. 23 (1944), 37-63.

[7] O’Neill, B., Elementary Differential Geometry, Academic Press, 1966.

[8] Website: www.mathworks.com. 